\title{
The laryngeal mask
}

\section{Useful for spontaneous breathing, controlled ventilation, and difficult intubations}

Obstruction of the upper airway is the commonest complication of general anaesthesia and is potentially life threatening. The first skill that all anaesthetists must therefore acquire is how to keep the airway patent. A further complication of anaesthesia is the loss of upper airway reflexes, which may result in the accidental aspiration of gastric contents into the tracheobronchial tree with the risk of extensive lung injury. To avoid these complications anaesthetists have developed several techniques, most notably tracheal intubation.

First used for anaesthesia in $1878,{ }^{1}$ this procedure has its problems - it often requires neuromuscular blockade, stimulates unwanted reflex activity, and may damage the vocal cords and tracheal mucosa. An alternative method, in starved patients who are breathing spontaneously, is to use the traditional facemask with or without an oropharyngeal (Guedel) airway. But even fasting patients may aspirate their gastric contents, and the Guedel airway does not invariably solve the problem of upper airway obstruction. Why this occurs during anaesthesia is unknown. Although the design of the Guedel airway was based on the assumption that the cause of upper airway obstruction was the tongue falling back, this is no longer believed to be the sole or even the main cause of obstruction. A more likely explanation is that a reduction in tone of pharyngeal structures results in obstruction at several locations. ${ }^{2-4}$

There are problems with using the anaesthetic facemask. The facial characteristics of individual patients, particularly those with beards or without teeth, do not always conform to the relatively uncompromising shape of traditional facemasks. In comparison with the tracheal tube there is more difficulty maintaining a seal and considerable fatigue in holding the mask for long periods. In addition, the anaesthetist does not have both hands free to attend to any complications that may arise during anaesthesia.

With these problems in mind Dr A I J Brain developed a new approach: the anaesthetic mask, instead of being applied to the face, was reduced in size so that it could be positioned over the laryngeal opening itself. ${ }^{5}$ Brain made plaster casts of the cadaveric pharynx to explore the space around the glottis. This boatshaped space inspired him to detach the black rubber cuff from a Goldman dental mask and draw it into the shape of an inflatable rubber boat and attach it to a tracheal tube. This first prototype, used in 1981, was inserted blindly under deep halothane anaesthesia. A satisfactory airway was immediately obtained, and inflating the lungs with gentle positive pressure ventilation was possible.

Seventy prototypes and several thousand patients later, commercial interest was aroused and the Dunlop Rubber Company made some latex and later silicone masks to the inventor's specifications. The first independent clinical trial was carried out at Northwick Park Hospital in $1987,{ }^{\circ}$ and within a year the design had been finalised and four sizes were available. By September 1990 all British hospitals performing operations had ordered the laryngeal mask, and it is now used extensively throughout the world.

The role of the laryngeal mask is threefold. It provides a patent airway for spontaneous breathing, an airway during controlled ventilation, and an alternative approach in patients who are difficult to intubate. In contrast to a conventional facemask the laryngeal mask fits around the upper end of the larynx as it protrudes into the pharynx. This results in a reduced requirement for fresh gas flow, allows more effective scavenging, facilitates monitoring of end tidal carbon dioxide concentration, and frees the anaesthetist's hands. It reduces the chances of aspiration of liquid from the upper airway, ${ }^{7}$ and for this reason the inventor advises that at the end of the anaesthetic the laryngeal mask should be kept in place until the patient starts to swallow, so that protection is maintained into the recovery period.

Use of the laryngeal mask as an alternative to tracheal intubation is more controversial. It is easier to insert than a tracheal tube, ${ }^{8}$ neuromuscular blockade is not necessary for its insertion, and controlling the airway is possible during placement. It is inserted blindly and, as with tracheal intubation, requires an adequate depth of anaesthesia during insertion to prevent excitatory phenomena such as coughing and laryngospasm. Satisfactory placement can usually be achieved after induction of anaesthesia with propofol but not with thiopentone alone. The haemodynamic response to insertion and the changes in intraocular pressure are less than those after placement of a tracheal tube. ${ }^{9}$ If gastric dilatation is to be avoided during controlled ventilation it is necessary to use smaller tidal volumes and avoid airway pressures greater than $25 \mathrm{~cm} \mathrm{H}_{2} \mathrm{O} .{ }^{10}$ The use of end tidal carbon dioxide and tidal volume monitors is advised.

Incorrect positioning of the laryngeal mask is not always clinically obvious, and the only certain way of ensuring correct positioning is by fibreoptic laryngoscopy. The cuff is permeable to nitrous oxide, and masks that initially seem satisfactorily positioned may become dislodged as the cuff inflates further. The commonest problem seems to be folding of the epiglottis, which is pushed down by the mask and partially obstructs the larynx. This complication occurs more commonly in children than adults ${ }^{11}$ and was recognised early in the development of the mask. The present shape is a compromise between the features facilitating successful placement and those providing an effective seal. The inventor emphasises the necessity for strict adherence to the protocol for insertion.

Many case reports have endorsed use of the laryngeal mask in difficult or impossible intubation. ${ }^{12}{ }^{13}$ As well as providing an airway it acts as a conduit through which a $6 \mathrm{~mm}$ internal diameter cuffed tracheal tube, gum elastic bougie, or fibreoptic laryngoscope can be passed. Although Brain does not endorse its use when aspiration of gastric contents is possible, reports of its use in obstetric anaesthesia have led some to suggest it should be considered as an alternative to emergency cricothyroidotomy if intubation has failed. Although studies in cadavers have shown that cricoid pressure may be applied effectively when a laryngeal mask is used, ${ }^{14}$ others are more cautious and suggest that the laryngeal mask may preferentially direct gastric contents towards the lungs. ${ }^{15}$ Ansermino and Blogg have shown that cricoid pressure may prevent insertion of the laryngeal mask, ${ }^{16}$ and removal of cricoid pressure or unsuccessful placement of the laryngeal mask may precipitate aspiration of gastric contents. Despite this problem the laryngeal mask may prove life saving when the patient is impossible to intubate or to ventilate by traditional methods.

Until recently the mainstay of upper airway patency was the anaesthetist grappling with the jaw and facemask. Astonishingly, more than 100 years after the introduction of endotracheal anaesthesia a new invention, developed in Britain by 
a determined, single minded anaesthetist, has transformed airway management and been adopted throughout the world.

MOIRA E O'MEARA

Senior Registrar

J GARETH JONES

Cambridge University Department of Anaesthesia,

Addenbrooke's Hospital,

Cambridge CB2 2QQ

1 Brandt L. The first reported oral intubation of the human trachea. Anesth Analg 1987;66:1198-9.

2 Drummond GB. Keep a clear airway. Br $\mathcal{f}$ Anaesth 1991;66:153-6.

3 Nandi PR, Charlesworth CH, Taylor SJ, Nunn JF, Dore CJ. Effect of general anaesthesia on the pharynx. Br f Anaesth 1991;66:57-162.

4 Hoffstein V, Zamel N. Sleep apnoea and the upper airway. Br F Anaesth 1990;65:139-50,

5 Brain AIJ. The development of the laryngeal mask. A brief history of the invention, early clinical studies and experimental work from which the laryngeal mask evolved. Eur f Anaesthesiol 1991; suppl 4:5-17.

6 Broderick PM, Webster NR, Nunn JF. The laryngeal mask airway. A study of 100 patients during spontaneous breathing. Anaesthesia 1989;44:1014-5.

7 John RE, Hill S, Hughes TJ. Airway protection by the laryngeal mask. A barrier to dye placed in the pharynx. Anaesthesia 1991;46:366-7.

8 Davies PR, Tighe SQ Greenslade GL, Evans GH. Laryngeal mask airway and tracheal tube insertion by unskilled personnel. Lancet 1990;336:977-9.

Watcha MF, White PF, Tychsen L, Stevens JL. Comparative effects of laryngeal mask airway and endotracheal tube insertion on intraocular pressure in children. Anesth Analg 1992;75:355-60.

10 Benumof JL. Laryngeal mask airway. Indications and contraindications. Anesthesiology 1992;77: 843-6.

11 Rowbottom SJ, Simpson DL, Gabb D. The laryngeal mask airway in children, a fibreoptic assessment of positioning. Anaesthesia 1991;46:489-91.

2 Beveridge ME. Laryngeal mask anaesthesia for repair of cleft palate. Anaesthesia 1989;44:656-7.

13 Heath ML, Allagain J. Intubation through the laryngeal mask. A technique for unexpected difficult intubation. Anaesthesia 1991;46:545-8.

14 Strang TI. Does the laryngeal mask airway compromise cricoid pressure? Anaesthesia 1992;47: $829-31$.

15 Nanji GM, Malty JR. Vomiting and aspiration pneumonitis with the laryngeal mask airway. Can $\mathcal{f}$ Anaesth 1992;39:69-70.

16 Ansermino JM Blogg CE. Cricoid pressure may prevent insertion of the laryngeal mask airway. $\mathrm{Br} \mathcal{F}$ Anaesth 1992;69:465-7.

\section{Diagnostic dilatation and curettage in young women}

\section{Should be replaced by outpatient endometrial biopsy}

A paper by Coulter and colleagues in this week's journal shows that although rates of hysterectomy are much higher in the United States than in Britain, dilatation and curettage is six times more common in Britain than the United States (p 236). ${ }^{1}$ When performed for diagnostic purposes few of these procedures in young women are likely to be justified.

Dilatation and curettage is diagnostically inaccurate and therapeutically ineffective. When performed on its own it will miss $10 \%$ of endometrial lesions ${ }^{2}$ : one study found that less than half the uterine cavity was curetted in more than half the patients. $^{3}$ Yet despite these long established defects of the procedure most British hospitals continue to admit young women with menstrual irregularities for diagnostic curettage. How should they be managing these patients instead?

In young women with oligomenorrhoea, or those with secondary amenorrhoea who are not pregnant the common diagnoses are post-pill amenorrhoea, polycystic ovarian disease, and disorders of the hypothalamic-pituitary axis, often associated with weight gain or weight loss. The diagnosis should be made by clinical examination supplemented by assays of steroid hormones, particularly testosterone, oestradiol, follicle stimulating hormone, and luteinising hormone. The ratio of follicle stimulating hormone to luteinising hormone changes in polycystic ovarian disease. A normal serum prolactin concentration excludes a pituitary microadenoma.

Young women with intermenstrual bleeding should first be questioned about their contraceptive technique as intrauterine contraceptive devices and oral contraceptives, particularly progestogen only pills, are commonly responsible. Local causes such as cervical polyps, chronic cervicitis, and, less commonly, cervical squamous cell carcinoma can almost always be detected by a speculum examination with a good light supplemented by cervical smear testing, colposcopy, and biopsy. Only 5\% of cervical cancers are endocervical adenocarcinomas and thus not visible on speculum examination. ${ }^{4}$ Intermenstrual bleeding due to intrauterine causes such as a mucous polyp is much less common, and cystic glandular hyperplasia or adenomatous hyperplasia is rare in women under 40 and can be diagnosed only by biopsy.

Although in women with subfertility it is often helpful to obtain a sample of endometrium in the second half of the menstrual cycle to confirm secretory changes, the presence of a raised serum progesterone concentration in the second half of the cycle is good evidence of ovulation. Obtaining an endometrial biopsy specimen at the time of laparoscopic assessment of tubal patency would, however, be reasonable.

Most women under 40 with menstrual problems present with heavy frequent periods, and most of them suffer from dysfunctional bleeding, with no gross pelvic disease. In these women endometrial adenocarcinoma is rare, and premalignant adenomatous hyperplasia is also extremely uncommon. Less than $1 \%$ of adenocarcinomas of the corpus uteri occur in women under 35 and only $6 \%$ occur in women up to the age of $45.5^{5}$ When they occur they are commonly a well differentiated hormone dependent type. ${ }^{4}$ Menstrual irregularities combined with dysmenorrhoea should always raise the possibility of a submucous fibroid polyp or adenomyosis, often associated with endometriosis. Ample evidence now exists, however, that a diagnostic curettage will not detect many submucous fibroids, and there is general agreement that hysteroscopy is the diagnostic method of choice. ${ }^{6-8}$ Dysfunctional bleeding is often self limiting or responds to a short course of cyclical steroids. Some gynaecologists still believe that diagnostic curettage has a therapeutic effect in these women, although many recent publications show no therapeutic advantage at all..$^{9}$

Why then does diagnostic curettage continue to be widely performed in Britain when there is ample evidence that an endometrial biopsy specimen taken in the clinic by endometrial aspiration provides sufficient tissue for accurate pathological diagnosis and exclusion of major endometrial disease? Many devices allow endometrial biopsy, including the Karman catheter, the Novak curette, the Vabra aspirator, the Gravlee Jetwasher, the endocyte device, ${ }^{11}$ and, more recently, the Pipelle De Cornier. ${ }^{12}$ The Pipelle De Cornier is made of soft plastic and can be inserted through the cervical os with less pain and discomfort than most of the other devices; being flexible, it easily traverses the cervical canal and is therefore ideal for outpatient use. Although hysterosalpingography and endovaginal scanning will diagnose submucous fibroid polyps, hysteroscopy, particularly in the proliferative phase of the cycle, is the diagnostic method of choice.

Not only is dilatation and curettage inaccurate in diagnosing uterine disease but it also risks trauma, especially in women who have not had children and therefore have an undilated cervix. Trauma may result in an incompetent cervix from excessive dilatation, perforation into the parametrium and broad ligament with formation of haematoma, and perforation of the fundus of the uterus with intraperitoneal bleeding. 